\title{
Geographies of Ageing: Social Processes and the Spatial Unevenness of Population Ageing
}

\author{
by Amanda Davies and Amity James \\ Surrey (UK): Ashgate Publishing 2011 \\ ISBN 978-1-40941-776-7 \\ Hardcover, \$109.95, 209+xiii pp.
}

\author{
Reviewed by Herbert C. Northcott \\ Department of Sociology, University of Alberta
}

Aged persons make up an increasing percentage of populations around the world. The major drivers of this trend are declines in fertility and mortality. With fewer children being born and more people surviving into old age, the percentage of seniors increases in the population. The authors note that this population aging trend is ongoing and will continue well into the 21 st century, creating an unprecedented shift in age structures worldwide. Nevertheless, the authors note that population aging is variable across various geographical units. That is, there is a spatial variability in population aging. This book explores the reasons for the spatial "unevenness" of population aging, and the implications of these patterns.

The authors situate their book as a contribution to the sub-discipline of population geography, which is concerned with the spatial aspects of demographic trends. The book is organized into eleven chapters, grouped into three parts that examine the spatial nature, the determinants, and the implications of population aging.

The authors begin by examining population aging as a global phenomenon with spatial variation across regions and countries. At this geographic level, population aging is primarily a function of fertility and mortality rates. The authors then examine spatial aging within countries by focusing on the case of Australia. The authors note that the distribution of the aging population within a country tends to be influenced by migration patterns. For example, out-migration of young adults from rural agricultural areas tends to increase the concentration of seniors who are more likely to stay in these rural areas. On the other hand, in-migration of young adults to cities tends to decrease the concentration of seniors aging in place in the cities.

Nevertheless, the authors point out that there are spatial variations in aging within cities. Seniors, for example, tend to be more concentrated in older neighborhoods, as they age in homes they purchased many years ago, while younger families tend to concentrate in the newer suburbs.

Further, the authors examine elder migration. The in-migration of seniors to certain lifestyle amenity regions, such as coastal areas near larger cities, illustrates the role that elder migration plays as a factor in the spatial variability of population aging. The authors also note that the international migration of seniors can be very consequential for certain popular retirement amenity destinations. For example, northern European seniors may choose to retire permanently or seasonally in various southern European countries. Both climate and economics are a factor, with seniors attracted to warmer climates and lower costs of living. A similar phenomenon is evident in North America, where Canadian seniors and seniors from the northern states in the USA often relocate to the warmer southern states, either permanently or seasonally.

Seniors who relocate are often already disengaged from the labour force, and therefore do not move for employment reasons. In contrast, the authors observe that the movement of younger people tends to be driven by education and employment opportunities. As noted previously, the movement of younger people is a major factor in the uneven distribution of the aging population within a country. Employment opportunities may be 
selective by social class, ethnicity, and gender as well as age, and therefore influence the spatial unevenness in the composition of the population not only by age but also by social class, ethnicity, and gender.

The authors examine seniors' residential choices. Many seniors prefer not to move, but instead prefer to grow older in the homes and communities in which they have long resided. Some seniors move to be closer to services that they need or expect to need as they grow older. Concentrations of seniors result when seniors stay or move in and younger persons leave. The authors denote high concentrations of older persons as "naturally occurring retirement communities." The authors also note that planned retirement communities designed specifically for older residents are becoming increasingly common.

The authors examine residential mobility in the context of the life course. Moves motivated by education, employment, retirement, and declines in health reflect the life course, and result in spatial variation in the concentration of various age groups.

In the final section of the book, the authors examine the implications of spatial variation in the distribution of age groups, and of seniors in particular. In general, the authors note that population aging is expected to result in labor shortages. Nevertheless, the authors point out that the impact of population aging will be spatially variable, reflecting the uneven spatial distribution of age groups. The authors observe that an aging population and concentrations of seniors will influence the development and distribution of the labor supply. Seniors, for example, will generate employment in the healthcare industry.

Finally, the authors reflect on the environmental impacts of population aging and the uneven distribution of age groups. Seniors may reduce environmental impacts by driving less, for example. Seniors who concentrate in amenity areas may foster environmental conservation efforts, while at the same time generating pressures on the environment as retirement communities grow in size.

In summary, this book provides a comprehensive overview of the spatial nature of population aging, the determinants of the "unevenness" of population aging, and the diverse implications of the spatial nature of population aging. 